\title{
DISENTANGLING CROSSCUTTING IN AOSD: FORMALIZATION BASED ON A CROSSCUTTING PATTERN*
}

\author{
José M. Conejero ${ }^{1}$, Klaas van den Berg ${ }^{2}$ and Juan Hernández ${ }^{1}$ \\ 1: Quercus Software Engineering Group \\ University of Extremadura \\ Avda. Universidad s/n C.P. 10071 Cáceres, Spain \\ e-mail: \{chemacm,juanher\}@unex.es web: http://quercusseg.unex.es \\ 2: Software Engineering Group \\ University of Twente \\ P.O. Box 217, 7500 AE Enschede, the Netherlands \\ e-mail: k.g.vandenberg@ewi.utwente.nl,web: http://trese.cs.utwente.nl/
}

Keywords: Aspect-Oriented Software Development, Scattering, Tangling, Crosscutting

\begin{abstract}
Crosscutting is usually described in terms of scattering and tangling. However, the distinction between these concepts is vague, which could lead to ambiguous statements. Sometimes, precise definitions are required, e.g., for the formal identification of crosscutting concerns. We propose a conceptual framework for formalizing these concepts based on a crosscutting pattern that shows the mapping between elements at two levels, e.g., concerns and representations of concerns. The definitions of the concepts are formalized in terms of linear algebra, and visualized with matrices and matrix operations. In this way, crosscutting can be clearly distinguished from scattering and tangling. The usability of dependency matrices is illustrated in the analysis of crosscutting across several refinement levels, which can be formalized through the cascading of the crosscutting pattern.
\end{abstract}

\section{INTRODUCTION}

One of the key principles in Aspect-Oriented Software Development (AOSD) is Separation of Concerns (SOC). A concern can be defined very generally as a thing in an engineering process about which it cares [7]. Related with this principle is the problem of crosscutting concerns. Crosscutting is usually described in terms of scattering and tangling, e.g., crosscutting is the scattering and tangling of concerns arising due to poor support for their modularization. However, the distinction between these concepts is vague, sometimes leading to ambiguous statements and confusion, as stated in [11]:

.. the term "crosscutting concerns" is often misused in two ways: To talk about a single concern, and to talk about concerns rather than representations of concerns. Consider "synchronization is a crosscutting concern": we don't know that synchronization is crosscutting unless we know what it crosscuts. And there may be representations of the

\footnotetext{
* In conjunction with AOSD-Europe Project IST-2-004349-NoE (see [1]) and MEC TIN2005-09405-C02-02
} 
concerns involved that are not crosscutting.

When talking about aspect orientation, we use these concepts for which we have some intuition based on our specific experience. However, precise definitions are mandatory in order to allow tool support, e.g., for mining of (crosscutting) concerns (c.f. [10]). The goal of this paper is to describe a conceptual framework with precise definitions of scattering, tangling and crosscutting.

The paper is structured as follows. In Section 2, we introduce the crosscutting pattern with definitions about crosscutting, tangling and scattering In Section 3 we describe how to represent and visualize crosscutting by means of matrices. In Section 4 , we discuss the cascading of crosscutting patterns. Section 5 shows the application of framework in a case study. Finally in Sections 6 and 7, we present related work and conclusions of the paper.

\section{CROSSCUTTING PATTERN}

In this section, we describe the assumption for the crosscutting pattern and the pattern itself. Furthermore, we focus on definitions of crosscutting, tangling and scattering.

\subsection{Generalization}

Our proposition is that tangling, scattering and crosscutting can only be defined in terms of 'one thing' with respect to 'another thing': at least two levels (or two domains or two phases) are related with each other in some way. For example:

- The term domain could refer to on one-hand concerns and on the other-hand representations of concerns [11], as stated in the citation in the introduction.

- The term level could refer to refinements in the Model Driven Architecture [14] (e.g. CIM, PIM and PSM).

- The term phase could refer to phases in the software development cycle, such as concern modelling, requirements analysis, architectural design, detailed design and implementation.

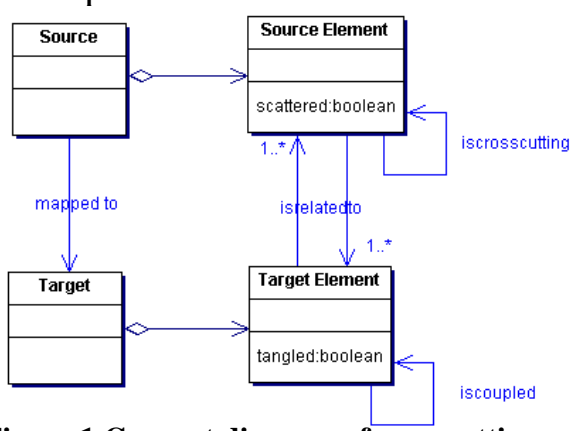

Figure 1 Concept diagram of crosscutting pattern

We use here the general terms source and target (as in [14]) to denote two consecutive phases, levels or domains (see Figure 1). We use the term pattern as in design patterns [9], in the sense of being a general description of frequently encountered situations [13], [15]: e.g. we have phrases such as "one thing with respect to another thing".

In the Crosscutting Pattern, elements in the source are related to elements in the target: there is a mapping between source and target elements. The mapping can be established manually or be automated in transformation rules. This mapping can have different types, such as usage and abstraction dependencies (e.g. refinement and tracing [17]). In this paper, we will not discuss intra-level (coupling) relations between elements. The terms crosscutting, tangling and scattering are defined as specific cases of these mappings. 


\subsection{Concepts based on Crosscutting Pattern}

As we can see in Figure 1, there is a mapping from source elements to target elements. Moreover, this mapping from source to target has a multiplicity. In case of 1:many mappings we have scattering, defined as follows: Scattering occurs when, in a mapping between source and target, a source element is related to multiple target elements. The correspondence between two given domains, Source and Target, is defined as follows: For element $s \epsilon$ Source, $f(s)=\{t \in$ Target / element $t$ is related to $s\}$. We can define scattering as: An element $s \epsilon$ Source is scattered if and only if card $(f(s))>1$, where card is the cardinality.

Similarly, we can focus on the relation between target elements and source elements. This relation (here also called mapping) is the reverse of the mapping above. In case of 1:many mappings from target to source we have tangling, defined as follows: Tangling occurs when, in a mapping between source and target, a target element is related to multiple source elements. In other words, an element $t \epsilon$ Target is tangled if and only if $\operatorname{card}\left(f^{1}(t)\right)>1$, where $f^{l}$ is the inverse application.

There is a specific combination of scattering and tangling which we call crosscutting, defined as follows: Crosscutting occurs when, in a mapping between source and target, a source element is scattered over target elements and where in at least one of these target elements, some other source elements are tangled. In other words, crosscutting can be defined as follows. For element s1, s2 $€$ Source, s1 crosscuts $s 2$ (with $s 1$ different from $s 2$ ) if and only if $\operatorname{card}(f(s 1))>1$ and $\exists t \epsilon f(s 1): \operatorname{card}\left(f^{1}(t)\right)>1$ and $s 2 \epsilon f^{1}(t)$. We do not require that the second source element is scattered. In that sense, our definition is not symmetric as definition in [13] (see Section 6).

\section{MATRIX REPRESENTATION}

In terms of linear algebra, the relation or mapping between source elements and target elements can be represented in a matrix that we called dependency matrix. A dependency matrix (source $x$ target) represents the dependency relation between source elements and target elements (inter-level relationship). In the rows, we have the source elements, and in the columns, we have the target elements. In this matrix, a cell with 1 denotes that the source element (in the row) is mapped to the target element (in the column). Reciprocally this means that the target element depends on the source element. Scattering and tangling can easily be visualized in this matrix. In Table 1, we can see an example of dependency matrix where S denotes a scattered source element - a grey row; NS denotes a non-scattered source element; T denotes a tangled target element - a grey column; NT denotes a non-tangled target element.

\begin{tabular}{|c|c|c|c|c|c|}
\hline & \multicolumn{4}{|c|}{ target } \\
\hline & & $\mathrm{t}[1]$ & $\mathrm{t}[2]$ & $\mathrm{t}[3]$ & $\mathrm{t}[4]$ \\
\hline \multirow{3}{*}{ 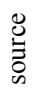 } & $\mathrm{s}[1]$ & 1 & 1 & 0 & 1 \\
\hline & $\mathrm{s}[2]$ & 0 & 0 & 1 & 0 \\
\hline & $\mathrm{s}[3]$ & 0 & 0 & 1 & 0 \\
\hline
\end{tabular}

\begin{tabular}{|c|c|c|c|c|}
\hline & cro & \multicolumn{3}{|c|}{ source } \\
\hline & & $\mathrm{s}[1]$ & $\mathrm{s}[2]$ & $\mathrm{s}$ [3] \\
\hline \multirow{3}{*}{ 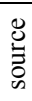 } & $\mathrm{s}[1]$ & 0 & 0 & 0 \\
\hline & $\mathrm{s}[2]$ & 0 & 0 & 0 \\
\hline & $\mathrm{s}[3]$ & 0 & 0 & 0 \\
\hline
\end{tabular}

Table 1. Example dependency and crosscutting matrix with tangling, scattering and no crosscutting

We define a new auxiliary concept crosscutpoint used in the context of dependency diagrams, to denote a matrix cell involved in both tangling and scattering. If there are one or more 
crosscutpoints then we say we have crosscutting.

Crosscutting between source elements for a given mapping to target elements can be represented in a crosscutting matrix. A crosscutting matrix (source $x$ source) represents the crosscutting relation between source elements, for a given source to target mapping (represented in a dependency matrix). In the crosscutting matrix, a cell with 1 denotes that the source element in the row is crosscutting the source element in the column. In Section 3.1 we explain how this crosscutting matrix can be derived from the dependency matrix. In Table 1, we can observe the crosscutting matrix for dependency matrix shown in the same table. In this example, we have one scattered source element $s[1]$ and one tangled target element $t[3]$. However, $\mathrm{t}[3]$ is not involved in the scattering of $\mathrm{s}[1]$. We apply our definition of crosscutting and arrive to the crosscutting matrix. In this case, there is no crosscutting.

Another example mapping is shown in Table 2. Here, we have one scattered source element $\mathrm{s}[1]$ and one tangled target element $\mathrm{t}[3]$. Moreover, there is one crosscutpoint at matrix cell [1,3] (dark grey cell). Again applying our definition, we arrive to the crosscutting matrix. Source element $s[1]$ is crosscutting $s[3]$ (because $s[1]$ is scattered over [t[1], t[3], t[4]] and $\mathrm{s}[3]$ is in the tangled one of these elements, namely t[3]. The reverse is not true: the crosscutting relation is not symmetric. This illustrates our proposition about crosscutting not being symmetric: $s[1]$ is crosscutting $s[3]$, but $s[3]$ is not crosscutting $s[1]$ because $s[3]$ is not scattered (scattering is a necessary condition for crosscutting).
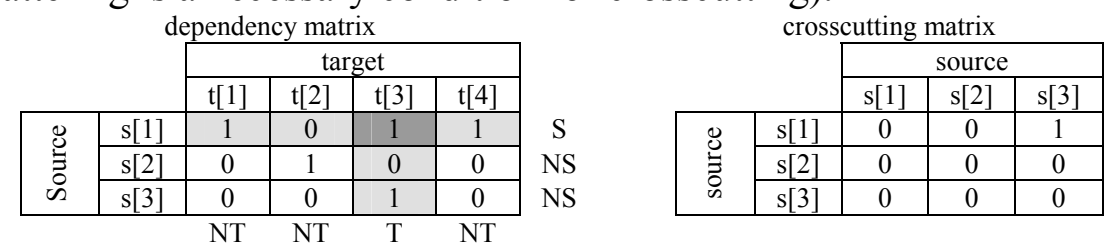

Table 2. Example dependency and crosscutting matrix with tangling, scattering and one crosscutting

\subsection{Constructing crosscutting matrices}

We now describe how to derive the crosscutting matrix from the dependency matrix. We use the example detailed in Table 2 of Section 3 to show the construction of crosscutting matrix. Based on the dependency matrix, we define some auxiliary matrices: the scattering matrix (source $\mathrm{x}$ target), and the tangling matrix (target $\mathrm{x}$ source). For our example in Table 2, these matrices are shown in Table 3.

\begin{tabular}{|c|c|c|c|c|c|}
\multicolumn{2}{c|}{ scattering matrix } \\
\cline { 3 - 6 } \multicolumn{2}{c|}{} & \multicolumn{4}{|c|}{ target } \\
\cline { 3 - 6 } \multicolumn{2}{c|}{} & $\mathrm{t}[1]$ & $\mathrm{t}[2]$ & $\mathrm{t}[3]$ & $\mathrm{t}[4]$ \\
\hline \multirow{E}{*}{} & $\mathrm{s}[1]$ & 1 & 0 & 1 & 1 \\
\cline { 2 - 6 } & $\mathrm{s}[2]$ & 0 & 0 & 0 & 0 \\
\cline { 2 - 6 } & $\mathrm{s}[3]$ & 0 & 0 & 0 & 0 \\
\hline
\end{tabular}

\begin{tabular}{|c|c|c|c|c|}
\hline & \multicolumn{3}{|c|}{$\frac{\text { tangling matrix }}{\text { source }}$} \\
\hline & & $\mathrm{s}[1]$ & $\mathrm{s}[2]$ & $\mathrm{s}$ S[3] \\
\hline \multirow{4}{*}{$\begin{array}{l}\overrightarrow{.} \\
\text { D. } \\
\text { t }\end{array}$} & $\mathrm{t}[1]$ & 0 & 0 & 0 \\
\hline & $\mathrm{t}[2]$ & 0 & 0 & 0 \\
\hline & $\mathrm{t}[3]$ & 1 & 0 & 1 \\
\hline & $\mathrm{t}[4]$ & 0 & 0 & 0 \\
\hline
\end{tabular}

Table 3. Scattering and tangling matrices for dependency matrix in Table 2

These two matrices are defined as follows:

- In the scattering matrix a row contains only dependency relations from source to target elements if the source element in this row is scattered (mapped onto multiple target elements); otherwise the row contains just zero's (no scattering). 
- In the tangling matrix a row contains only dependency relations from target to source elements if the target element in this row is tangled (mapped onto multiple source elements); otherwise the row contains just zero's (no tangling).

We now define the crosscutting product matrix, showing the frequency of crosscutting relations. A crosscutting product matrix (source $x$ source) represents the frequency of crosscutting relations between source elements, for a given source to target mapping. The crosscutting product matrix is not necessarily symmetric. The crosscutting product matrix ccpm can be obtained through the matrix multiplication of the scattering matrix sm and the tangling matrix tm: $c c p m=s m . t m$ where ccpm $[i][k]=s m[i][j] \mathrm{tm}[j][k]$.

In this crosscutting product matrix, the cells denote the frequency of crosscutting. This can be used for quantification of crosscutting (crosscutting metrics). In this example, there are no cells in the crosscutting product matrix larger than 1 . A cell on the diagonal which contains 1 denotes a crosscutting relation with itself, which we disregard here. In the crosscutting matrix, we put the diagonal cells to 0. Obviously, this is because we assume that a source element can not crosscut itself.

\begin{tabular}{|c|c|c|c|c|}
\hline \multicolumn{4}{|c|}{ crosscutting product matrix } & source \\
\hline & & $\mathrm{s}$ [1] & $\mathrm{s}[2]$ & $\mathrm{s}[3]$ \\
\hline \multirow{3}{*}{ 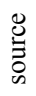 } & $\mathrm{s}[1]$ & 1 & 0 & 1 \\
\hline & $\mathrm{s}[2]$ & 0 & 0 & 0 \\
\hline & $\mathrm{s}[3]$ & 0 & 0 & 0 \\
\hline
\end{tabular}

\begin{tabular}{c|c|c|c|c|}
\multicolumn{4}{c|}{ crosscutting matrix } \\
\cline { 3 - 5 } \multicolumn{2}{c|}{} & \multicolumn{3}{|c|}{ source } \\
\cline { 2 - 5 } \multicolumn{2}{c|}{} & $\mathrm{s}[1]$ & $\mathrm{s}[2]$ & $\mathrm{s}[3]$ \\
\hline \multirow{\Xi}{*}{} & $\mathrm{s}[1]$ & 0 & 0 & 1 \\
\cline { 2 - 5 } & $\mathrm{s}[2]$ & 0 & 0 & 0 \\
\cline { 2 - 5 } & $\mathrm{s}[3]$ & 0 & 0 & 0 \\
\hline
\end{tabular}

Table 4. Crosscutting product matrix and crosscutting matrix for dependency matrix in Table 2

In the crosscutting matrix, a matrix cell denotes the occurrence of crosscutting; it abstracts from the frequency of crosscutting. The crosscutting matrix ccm can be derived from the crosscutting product matrix ccpm using a simple conversion: : $c c m[i][k]=$ if $(c c p m[i][k]>0)$ $\wedge(i \neq k)$ then 1 else 0 . The crosscutting product matrix and the crosscutting matrix for the example are given in Table 4. For convenience, these formulas can be calculated automatically by means of simple mathematic tools. By filling in the cells of the dependency matrix, the other matrices are calculated automatically.

\section{CASCADING OF CROSSCUTTING PATTERNS}

Usually we encounter a number of consecutive levels or phases in software development. In MDA [14], we have transformations from Platform Independent Models, Platform Specific Models to Implementation Models. From the perspective of software life cycle phases, we could distinguish Domain Analysis, Concern Modelling, Requirement Analysis, Architectural Design, Detailed Design, and Implementation.

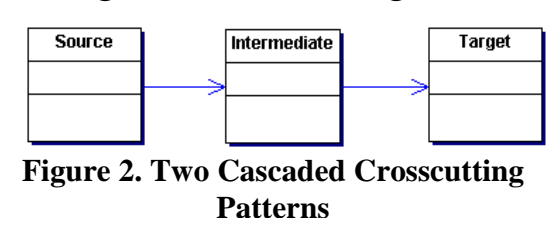

We consider here the cascading of two crosscutting patterns: the target of the first pattern serves as source for the second one. We call the first target our intermediate level, and the second target just target (Figure 2). Each of these mappings can be described with a dependency matrix. We describe how to combine two consecutive dependency matrices, in an operation we call cascading. Cascading is an operation on two dependency matrices resulting in a new dependency matrix, which represents the dependency relation between source elements of the first matrix and 
target elements of the second matrix.

For cascading, it is essential to define the transitivity of dependency relations. Transitivity is defined as follows. Assume we have a source, an intermediate, and a target level. There is a dependency relation between an element in the source and an element in the target if there is some element at the intermediate level that has a dependence relation with this source element and a dependency relation with this target element. In other words, the transitivity dependency relation $\mathrm{R}$ for source $\mathrm{s}$, intermediate level $\mathrm{u}$ and target $\mathrm{t}$, and $\operatorname{card}(\mathrm{u})$ is the number of elements in $\mathrm{u}: \exists k \epsilon(1 . . u):(s[i] R u[k]) \wedge(u[k] R t[m]) \Rightarrow(s[i] R t[m])$

Cascading may also be generalized to n-levels through function composition [5].

As an example, we explain the cascading of two dependency matrices: one for concerns $\mathrm{x}$ requirements and one for requirements $\mathrm{x}$ modules. The two dependency matrices are shown in Table 5.

\begin{tabular}{|c|c|c|c|c|}
\cline { 2 - 5 } \multicolumn{1}{c|}{} & \multicolumn{4}{c|}{ requirement } \\
\hline concern & $\mathrm{r}[1]$ & $\mathrm{r}[2]$ & $\mathrm{r}[3]$ & $\mathrm{r}[4]$ \\
\hline $\mathrm{c}[1]$ & 1 & 0 & 0 & 1 \\
\hline $\mathrm{c}[2]$ & 0 & 1 & 0 & 0 \\
\hline $\mathrm{c}[3]$ & 0 & 0 & 1 & 1 \\
\hline
\end{tabular}

\begin{tabular}{|c|c|c|c|c|c|}
\hline \multirow[b]{2}{*}{ requirement } & \multicolumn{5}{|c|}{ module } \\
\hline & $\mathrm{m}[1]$ & $\mathrm{m}[2]$ & $\mathrm{m}[3]$ & $\mathrm{m}[4]$ & $\mathrm{m}[5]$ \\
\hline $\mathrm{r}[1]$ & 1 & 0 & 0 & 0 & 1 \\
\hline $\mathrm{r}[2]$ & 0 & 1 & 0 & 0 & 0 \\
\hline $\mathrm{r}[3]$ & 0 & 1 & 1 & 0 & 0 \\
\hline $\mathrm{r}[4]$ & 0 & 0 & 0 & 1 & 1 \\
\hline
\end{tabular}

Table 5. Dependency matrices of two cascaded patterns

The first dependency matrix relates concerns with requirements. The second dependency matrix relates requirements with modules. The resulting dependency matrix relates concerns with modules (see Table 6). This matrix can be used to derive the crosscutting matrix for concern $x$ concern with respect to modules.

\begin{tabular}{|c|c|c|c|c|c|}
\cline { 2 - 6 } \multicolumn{1}{c|}{} & \multicolumn{5}{c|}{ modulting dependency matrix } \\
\hline concern & $\mathrm{m}[1]$ & $\mathrm{m}[2]$ & $\mathrm{m}[3]$ & $\mathrm{m}[4]$ & $\mathrm{m}[5]$ \\
\hline $\mathrm{c}[1]$ & 1 & 0 & 0 & 1 & 2 \\
\hline $\mathrm{c}[2]$ & 0 & 1 & 0 & 0 & 0 \\
\hline $\mathrm{c}[3]$ & 0 & 1 & 1 & 1 & 1 \\
\hline
\end{tabular}

\begin{tabular}{|c|c|c|c|}
\cline { 2 - 4 } \multicolumn{4}{c|}{ crosscutting matrix } \\
\cline { 2 - 4 } \multicolumn{3}{c|}{ concern } \\
\hline $\mathrm{concern}$ & $\mathrm{c}[1]$ & $\mathrm{c}[2]$ & $\mathrm{c}[3]$ \\
\hline $\mathrm{c}[2]$ & 0 & 0 & 1 \\
\hline $\mathrm{c}[3]$ & 0 & 0 & 0 \\
\hline
\end{tabular}

Table 6. The resulting dependency matrix and crosscutting matrix based on cascading of the matrices in Table 5

From this description, it is clear that cascading can be used for traceability analysis across multiple levels, e.g. from concerns to implementation elements, via requirements, architecture and design (c.f.[5]).

\section{CASE STUDY}

In this section, we show the application of our approach in a case study. This case has been used for some workshops, e.g. [8]. The case study implements a Conference Review System (CRS) [6]. For space reasons, we have used a simplification of this system. The general purpose of the system is to assist a conference's program committee to perform the review of papers and registration of participants of such conference.

There are four different user types in the system: PcChair, PcMembers, Authors and Participants. A PcChair is the main responsible of the review process. He has access to every paper and every review in the system. A PcMember takes over the reviews of the papers. A PcMember can see information of the papers but not reviews by other PcMembers. An Author 
can submit papers to the system. An Author can see only information about his own submission. A Participant must register in order to attend the conference. The register process is completely separated from the login process. However, once a user has registered he will need log whenever he accesses the system. This login process checks the role of the user in the system. The use case model of the conference review system is shown in Figure 3 . The complete requirements' analysis can be seen in [6].

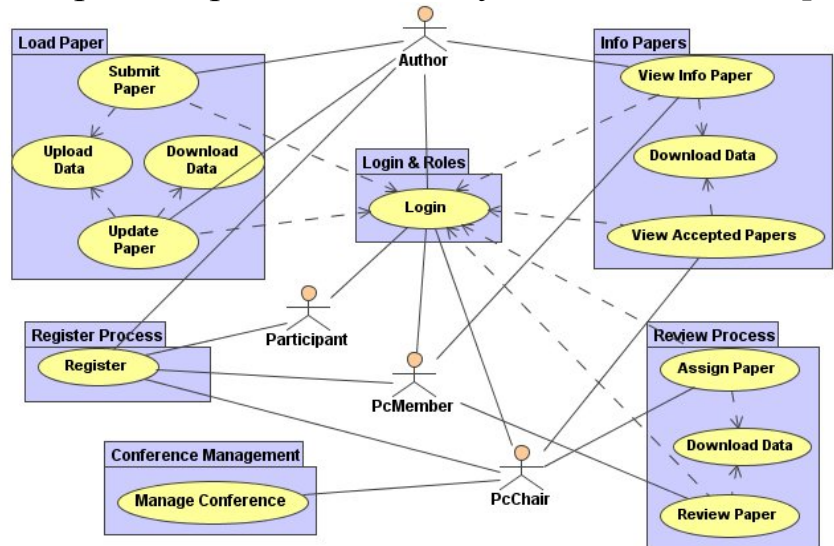

Figure 3. Use case model of the Conference Review System
We identified the following eight concerns: Papers Submission (PS), Papers Queries (PQ), Registration (Reg), Conference (C), Review (R), Information Retrieval/Supply (IRS), Login (L) and User Types (UT). Before applying our approach to identify crosscutting concerns in this example, a certain decomposition of requirements must be selected to analyze the mappings between concerns and artefacts of such a decomposition. Different decompositions can be performed, from coarse to fine grain. Here, we take the elements in the use case model (each package) shown in Figure 3 and the set of actors which take part in system as decomposition of requirements (coarse grain).

\begin{tabular}{|c|c|c|c|c|c|c|c|c|c|}
\hline & & \multicolumn{7}{|c|}{ Requirements } & \\
\hline & & \begin{tabular}{|l|} 
Register \\
Process
\end{tabular} & \begin{tabular}{|c|} 
Info \\
Papers
\end{tabular} & $\begin{array}{l}\text { Load } \\
\text { Papers }\end{array}$ & $\begin{array}{l}\text { Review } \\
\text { Process }\end{array}$ & $\begin{array}{l}\text { Conf. } \\
\text { Manag }\end{array}$ & $\begin{array}{l}\text { Login } \\
\& \text { Roles }\end{array}$ & Actors & \multirow[b]{2}{*}{ NS } \\
\hline \multirow{8}{*}{ 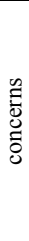 } & PS & 0 & 0 & 1 & 0 & 0 & 0 & 0 & \\
\hline & PQ & 0 & 1 & 0 & 0 & 0 & 0 & 0 & NS \\
\hline & Reg & 1 & 0 & 0 & 0 & 0 & 0 & 0 & NS \\
\hline & $\mathrm{C}$ & 0 & 0 & 0 & 0 & 1 & 0 & 0 & NS \\
\hline & $\mathrm{R}$ & 0 & 0 & 0 & 1 & 0 & 0 & 0 & NS \\
\hline & IRS & 1 & 1 & 1 & 1 & 1 & 0 & 0 & $\mathrm{~S}$ \\
\hline & $\mathrm{L}$ & 0 & 1 & 1 & 1 & 1 & 1 & 0 & $\mathrm{~S}$ \\
\hline & UT & 0 & 0 & 0 & 0 & 0 & 0 & 1 & NS \\
\hline
\end{tabular}

Table 7. (a) Dependency matrix concerns $x$ reqs and (b) crosscutting matrix for the Conference Review System

In Table $7 \mathrm{a}$ we show the dependency matrix with mappings between concerns and requirements and in Table $7 \mathrm{~b}$ the crosscutting matrix obtained from the former. Other decompositions of both concerns and requirements would be possible and consequently, the dependency matrix would be different. For example, we could use a finer grain decomposition of requirements such as each use case and each actor, thus increasing the size of tables. Anyway, the approach with a finer grain decomposition would be similar to the coarse grain decomposition shown in Table 7.

As we can see in Table $7 b$, the Login concern crosscuts every concern where the user must authenticate and system must check the role of such user. Similarly, the Information Retrieval/Supply concern crosscuts the concerns which need an access to the correspondence information to perform their actions.

Once we have identified the crosscutting concerns with respect to the requirements domain, 
we can observe how the concerns are related to the design of the system.

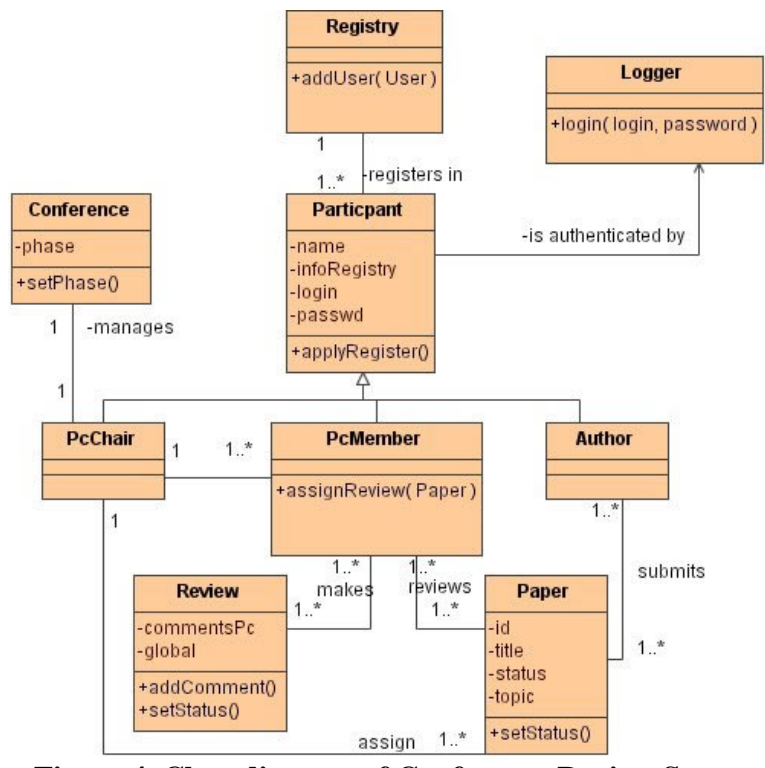

Figure 4. Class diagram of Conference Review System

We show in Figure 4 a simple UML class diagram representing the static structure of the design.

Now, we take the requirements as represented in the use case model as source elements, and the classes in the class diagram of the design as target elements.

We can build the dependency matrix shown in Table 8 to show the trace dependencies between requirements and design elements. As we can see in Table 8, the trace dependencies between Requirements and classes are direct mappings except for Register Process and Login\&Roles because of information added in the Participant class for such register and login purposes respectively (infoRegister and login, passwd attributes of Participant class). These requirements are tangled in such class with the own functionality of Participant class (User Type).

\begin{tabular}{|c|c|c|c|c|c|c|c|c|c|c|}
\hline \multicolumn{11}{|c|}{ asses } \\
\hline & & Pape & $\begin{array}{c}\text { Revie } \\
\mathrm{w}\end{array}$ & $\begin{array}{c}\text { Confer } \\
\text { ence }\end{array}$ & \begin{tabular}{|c|} 
Pc \\
Chair
\end{tabular} & $\begin{array}{c}\text { Pc } \\
\text { Member }\end{array}$ & Author & $\begin{array}{c}\text { Partic } \\
\text { ipant }\end{array}$ & $\begin{array}{c}\text { Logg } \\
\text { er }\end{array}$ & \begin{tabular}{|c|} 
Regis \\
try
\end{tabular} \\
\hline \multirow{7}{*}{ 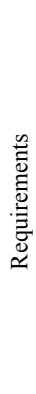 } & $\begin{array}{l}\text { Register } \\
\text { Process } \\
\end{array}$ & 0 & 0 & 0 & 0 & 0 & 0 & 1 & 0 & 1 \\
\hline & $\begin{array}{c}\text { Info } \\
\text { Papers }\end{array}$ & 1 & 0 & 0 & 0 & 0 & 0 & 0 & 0 & 0 \\
\hline & $\begin{array}{c}\text { Load } \\
\text { Papers }\end{array}$ & 1 & 0 & 0 & 0 & 0 & 0 & 0 & 0 & 0 \\
\hline & $\begin{array}{l}\text { Review } \\
\text { Process }\end{array}$ & 0 & 1 & 0 & 0 & 0 & 0 & 0 & 0 & 0 \\
\hline & $\begin{array}{c}\text { Conf. } \\
\text { Manag }\end{array}$ & 0 & 0 & 1 & 0 & 0 & 0 & 0 & 0 & 0 \\
\hline & $\begin{array}{c}\text { Login\& } \\
\text { Roles }\end{array}$ & 0 & 0 & 0 & 0 & 0 & 0 & 1 & 1 & 0 \\
\hline & Actors & 0 & 0 & 0 & 1 & 1 & 1 & 1 & 0 & 0 \\
\hline
\end{tabular}

Table 8. Dependencv matrix requirements $\mathrm{x}$ design

We apply the cascading operation (defined in Section 4) between the dependency matrix concerns $x$ requirements (Table 7a) and the dependency matrix requirements $\mathrm{x}$ design (Table 8) to obtain mappings between concerns and design elements. This derived dependency matrix concerns $\mathrm{x}$ design is shown in Table 9a. Finally, applying our definition of crosscutting to derived dependency matrix, we obtain the crosscutting matrix shown in Table 9b. From this matrix we can observe that with respect to the design -there are some additional crosscutting concerns (compared with Table 7b). The Registration concern (Reg) crosscuts the concerns Information Retieval/Supply (IRS), Login (L) and User Types (UT). Similarly, the concern UT crosscuts the concerns Reg, IRS and L. As we showed in dependency matrix obtained by means of the cascading operation (see Table 9a), these concerns are scattered in several design modules and in at least one of these modules other concern is tangled.

Obviously, this conclusion about crosscutting depends very much on the decomposition at each level and the dependencies between elements at these levels. There are many alternatives, which could aim at avoiding crosscutting by using another modularization (e.g. aspect-oriented techniques such as [3]). Here, we showed how to analyse crosscutting across 
several phases in the software life cycle.
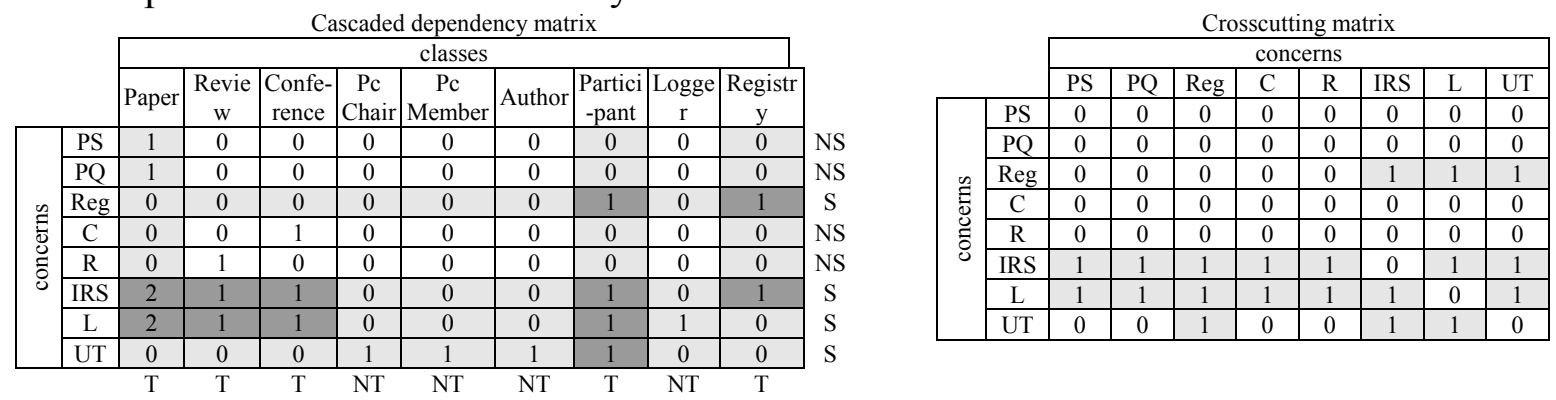

Table 9. Cascaded dependency matrix concerns $x$ design (9a) and crosscutting matrix (9b)

\section{RELATED WORK}

Some other publications have addressed the formalization of crosscutting, one of the core concepts in AOSD. In [13], crosscutting is defined as follows: "For a pair of modules $\mathrm{m}_{\mathrm{A}}$ and $\mathrm{m}_{\mathrm{B}}$ we say that $m_{A}$ crosscuts $m_{B}$ with respect to $X$ [the result domain] if and only if their projections onto $X$ intersect, and neither of the projections is a subset of the other." In our terminology, there is a source consisting of A and B and a target X. The intersection in this definition is similar to our definition of crosscutpoint (involved both in tangling and scattering). However, our definition is less restrictive because it does not require the subset relation: we only require that the cardinality of the projection of $\mathrm{m}_{\mathrm{A}}$ onto $\mathrm{X}$ is larger than 1 (scattering), but the cardinality of $\mathrm{m}_{\mathrm{B}}$ onto $\mathrm{X}$ can be larger or equal than 1 . This also implies that in our definition, crosscutting is not a symmetric property. The same remarks apply to a similar definition of crosscutting in [15].

Several authors use matrices (design structure matrices, DSM) to analyze modularity in software design [2]. In [12], a method is described with clustering and partitioning of the design structure matrix for improving modularity of object-oriented designs. However, the design structure matrices represent intra-level dependencies and not the inter-level dependencies as in the dependency matrices used for our analysis. In [16], a relationship matrix (concern $\mathrm{x}$ requirement) is described very similar to our dependency matrix, and used to identify crosscutting concerns. However, there is no explicit definition of crosscutting.

The papers described above lack an application of their definition of crosscutting to consecutive levels. We used our formalization to trace crosscutting concerns through different levels of a software development process, as shown by the cascading operation.

\section{CONCLUSIONS}

In this paper, we proposed a conceptual framework for describing crosscutting. We introduced a crosscutting pattern with a mapping between source elements onto target elements. With source and target, we abstract from specific levels or phases in software development. We defined crosscutting, tangling and scattering as separated cases based on specific mappings between source and target. We introduced the dependency matrix and crosscutting matrix to visualize the definitions. We showed that it is possible to formalize these definitions. 
The framework can be applied in concrete areas such as identification of crosscutting concerns in any phase of software life cycle [4] or the definition of crosscutting metrics, e. g. to quantify the intensity of crosscutting. Another interesting application is the cascading of crosscutting patterns, which can be used to model crosscutting relations across several levels, for example from concern modelling, to requirements, architectural design to detailed design and implementation. As such, it provides an approach for traceability analysis [5].

\section{REFERENCES}

[1] AOSD-Europe (2005). AOSD Ontology 1.0 - Public Ontology of Aspect-Orientation. Retrieved May, 2005, from http://www.aosd-europe.net/documents/d9Ont.pdf.

[2] Baldwin, C.Y. \& Clark, K.B. (2000). Design Rules vol I, The Power of Modularity. MIT Press.

[3] Baniassad, E. \& Clarke, S. (2004). Theme: An Approach for Aspect-Oriented Analysis and Design. In 26th International Conference on Software Engineering, Edinburgh.

[4] Berg, K. van den, Conejero, J. M. \& Hernández, J. (2006a). Identification of crosscutting in software design. In Aspect-Oriented Modeling Workshop at 5th AOSD, Bonn.

[5] Berg, K. van den, Conejero, J. M. \& Hernández, J. (2006b). Analysis of Crosscutting across Software Development Phases based on Traceability. In Early Aspects Workshop at 28th ICSE, Shanghai.

[6] Cachero. C., Gómez, J., Párraga, A. \& Pastor, O. (2001). Conference Review System: A Case of Study. In [8].

[7] Filman, R., et al. (2004). Aspect-Oriented Software Development. Addison-Wesley.

[8] First International Workshop on Web-Oriented Software Technology. (2001). http://www.dsic.upv.es/ west/iwwost01/ . Valencia.

[9] Gamma, E., Helm, R., Johnson, R., \& Vlissides, J. (1995). Design patterns. Elements of reusable objectoriented software. Addison-Wesley.

[10] Hannemann, J. \& Kiczales, G. (2001). Overcoming the Prevalent Decomposition in Legacy Code. In Workshop on Advanced Separations of Concerns, at ICSE May 2001, Toronto.

[11] Kiczales, G. Crosscutting. AOSD.NET Glossary 2005. At http://aosd.net/ wiki/index.php?title=Crosscutting.

[12] Lopes, C.V. and Bajracharya, S.K. (2005). An analysis of modularity in aspect oriented design. In 4th International Conference on Aspect-Oriented Software Development. 2005. Chicago.

[13] Masuhara, H. and Kiczales, G. (2003). Modeling Crosscutting in Aspect-Oriented Mechanisms. In 17th European Conference on Object Oriented Programming. 2003. Darmstadt.

[14] MDA (2003). MDA Guide Version 1.0.1, document number omg/2003-06-01.

[15] Mezini, M. and Ostermann, K. (2003). Modules for Crosscutting Models. In 8th International Conference on Reliable Software Technologies. Toulouse, France: LNCS 2655.

[16] Rashid, A., Moreira, A. and Araujo, J. (2003). Modularisation and Composition of Aspectual Requirements. In 2nd International Conference on Aspect Oriented Software Development. 2003. Boston.

[17] UML (2004). Unified Modeling Language 2.0 Superstructure Specification. At http://www.omg.org/cgi-bin/doc?ptc/2004-10-02 